\title{
Early Events in Herpes Simplex Virus Lifecycle with Implications for an Infection of Lifetime
}

\author{
Sarah Salameh ${ }^{1,3}$, Urmi Sheth ${ }^{1,3}$ and Deepak Shukla ${ }^{*, 1,2}$ \\ ${ }^{I}$ Department of Ophthalmology and Visual Sciences, College of Medicine, University of Illinois at Chicago, 60612, USA \\ ${ }^{2}$ Department of Microbiology and Immunology, College of Medicine, University of Illinois at Chicago, 60612, USA \\ ${ }^{3}$ Illinois Mathematics and Science Academy, 1500 Sullivan Rd., Aurora, IL 60506-1000, USA
}

\begin{abstract}
Affecting a large percentage of human population herpes simplex virus (HSV) types -1 and -2 mainly cause oral, ocular, and genital diseases. Infection begins with viral entry into a host cell, which may be preceded by viral "surfing" along filopodia. Viral glycoproteins then bind to one or more of several cell surface receptors, such as herpesvirus entry mediator (HVEM), nectin-1, 3-O sulfated heparan sulfate (3-OS HS), paired immunoglobulin-like receptor $\alpha$, and non-muscle myosin-IIA. At least five viral envelope glycoproteins participate in entry and these include $\mathrm{gB}, \mathrm{gC}, \mathrm{gD}$ and $\mathrm{gH}-\mathrm{gL}$. Post-entry, these glycoproteins may also facilitate cell-to-cell spread of the virus, which helps in the evasion of physical barriers as well as several components of the innate and adaptive immune responses. The spread may be facilitated by membrane fusion, movement across tight junctions, transfer across neuronal synapses, or the recruitment of actin-containing structures. This review summarizes some of the recent advances in our understanding of HSV entry and cell-to-cell spread.
\end{abstract}

Keywords: HSV, viral entry, glycoproteins, membrane fusion, viral surfing.

\section{INTRODUCTION}

The herpesviridae family consists of over 70 viral species, eight of which, herpes simplex virus-1 (HSV-1), herpes simplex virus-2 (HSV-2), varicella-zoster virus (VZV), cytomegalovirus (CMV), human herpesvirus 6 (HHV-6), human herpesvirus 7 (HHV-7), human herpesvirus 8 (HHV-8) and Epstein-Barr virus, are pathogenic to humans and may infect anywhere from thirty to over ninety percent of the adult human population [1]. Herpesviruses are characterized by linear, double-stranded DNA enclosed in icosahedral capsids. About 15-20 tegument proteins enclose the capsid, as well as a lipid bilayer membrane envelope derived from host cells [2]. All herpes viruses enter into latency after primary infection, establishing infection for the lifetime of their hosts. From their latency, herpesviruses can be reactivated, which has been correlated with a weakening of a host's immune defenses against the virus, to cause various diseases [3]. These diseases often involve viral shedding, or are contagious, allowing the spread of a specific herpesvirus within a population.

Affecting a significant majority of the general populace, herpes simplex virus 1 (HSV-1) represents one of the most studied herpesviruses. It causes mainly oral and ocular manifestations. Facial herpes causes an annual 500,000 cases of herpes labialis, HSV infection of the lips, mouth, gums or lip area [3]. After primary infection, HSV-1 enters periods of

*Address correspondence to this author at the Department of Ophthalmology and Visual Sciences (M/C 648), University of Illinois at Chicago, $1855 \mathrm{~W}$. Taylor Street, Chicago, IL 60612, USA; Tel: 312-355-0908;

E-mail:dshukla@uic.edu latency in the trigeminal or cervical ganglia, where it can be reactivated to cause recurrent episodes of viral shedding at the skin through lesions and sores. Asymptotic viral shedding can also occur, as it was reported in over $90 \%$ of adults and often the virus was shed without an indication of shedding through a lesion or sore [4]. One can contract HSV-1 through direct contact with sites of viral shedding, or with mucocutaneous fluids, which can also carry the virus [4]. Diseases caused by HSV-1 in the throat and mouth include pharyngitis, tonsillitis, and gingivostomatitis, which cause inflammation of pharynx and tonsils and swelling of the gums [2]. Ocular HSV-1 infections lead to several conditions, including stromal keratitis, blepharitis and retinitis, which are inflammations of the cornea, eyelid and retinarespectively [5]. In addition, immunocompromised hosts can suffer from HSV encephalitis, visceral infections including but not limited to pneumonitis, esophagitis, and hepatitis.

HSV-2 affected 45 million people aged 14 years or above in the United States alone and is the leading cause of recurrent genital herpes cases worldwide [6]. Genital herpes is characterized by painful ulcerated lesions and sores on the genitalia, although it can be asymptotic as well. Genital herpes increases the risk of contracting HIV by 2 or 3 times, as it is believed that $40-60 \%$ of HIV patients show prior infection with HSV-2 [6]. Schacker et al. repeatedly detected HIV RNA in genital ulcers, while they reported a decrease in HIV viral shedding with the healing of Genital Ulcer Disease (GUD) [7]. Recurrent genital herpes is mainly caused by HSV-2, as HSV-1 is less likely to be reactivated from the sacral ganglia, where HSV-2 latency and reactivation occurs [7]. Similar to HSV-1, HSV-2 can be contracted through direct contact with sites of viral shedding, which occurs after 
reactivation of the virus from its latency in sacral ganglia. Therefore, shedding of the virus from genital areas during sexual intercourse is the main method of contracting or spreading the virus [8].

However, there are additional methods of contracting HSV-2 as well. Recent studies have placed emphasis on the transfer of mainly HSV-2, but also HSV-1, from mother to child. The most common method of vertical transfer of HSV occurs during child birth through contact with an infected birth canal. Neonates can also contract HSV through contact with an HSV-infected caregiver or through ingestion of infected maternal secretions, known as intrauterine infections [9]. Neonatal infections by HSV-2 or HSV-1 pose serious threats, as they often lead to fatal diseases. These include SEM disease (disease located in the skin, eye or mouth), herpes encephalitis, or dispersed multi-organ infection [10]. HSV-2 can cause meningitis in neonates, which is usually more severe and life-threatening than Mollaret meningitis. The latter is a rare form of recurrent meningitis also caused by HSV-2 in adults. Unlike neonatal meningitis, it is aseptic, mild, and usually self-limiting.

HSV holds several factors that allow it to evade the immune system, leading to chronic infection in the body. HSV-1 glycoprotein $\mathrm{gC}$ binds $\mathrm{C} 3 \mathrm{~b}$ of the complement cascade, preventing the formation of anaphylatoxin $\mathrm{C} 5 \mathrm{a}$, as well as the formation of the Membrane attack complex (MAC) [11]. MAC is normally responsible for disrupting the phospholipid bilayer of infected cells, leading to cell lysis and death. Also, The HSV-1 gene ICP47 binds to the peptide transporter associated with antigen presentation, leading to the prevention of peptides on the major histocompatibility complex 1 (MHC1), thereby inhibiting the presentation of viral antigens to cytotoxic T-cells [12]. Other virulence factors include the $\mathrm{gE}$-gI heterodimer, as well as viral host shutoff protein (vhs). Vhs has several functions, which include the prevention of production of IFN- $\alpha$, which works with cytokines in intercellular signaling as well and plays a role in antiviral response, through the reduced IRF7 activation. Additionally, vhs is able to suppress proinflammatory cytokines and chemokines, inhibit antigen presentation by MHCI and MHCII, and inhibit the maturation of dendritic cells. The glycoproteins gE-gI have been shown to inhibit the activation of the complement cascade by binding to the Fc domain on $\mathrm{IgG}$ of antibodies which activate the classical complement cascade [13]. Methods by which the virus enter host-cells and the machinery that allow the virus to evade immune defenses, including cell-to-cell spread, help establish lifelong infection by HSV-1 and HSV-2.

\section{ENTRY}

HSV-1 and HSV-2 utilize several glycoprotein-receptor complexes when entering into host cells (Fig. 1). Both express the glycoproteins $\mathrm{gB}, \mathrm{gC}, \mathrm{gD}, \mathrm{gH}$, and $\mathrm{gL}$. All except $\mathrm{gC}$ are essential for host cell entry, although the absence of $\mathrm{gC}$ does reduce efficiency [1]. $\mathrm{gC}$ makes the first contact with the host cell, binding to heparan sulfate (HS) proteoglycans on the cell surface. In the absence of $\mathrm{gC}, \mathrm{gB}$ is able to carry out this function. HSV-1 has been shown to exercise several methods to attach to the host cell. In some cases, HSV-1 travels down filopodia-like membrane protrusions to reach the cell body for internalization. This extracellular transport of virions is called "surfing"(Fig. 1) [14]. Exposure to HSV-1 can induce the formation of these actin-rich, filopodia-like structures by the cell. This is facilitated through members of the Rho GTPase family, which have been shown to act as a link between surface receptors such as syndecans or integrins and the actin cytoskeleton organization underneath $[15,16]$. At the activation of these small GTPases, filopodia are formed. The glycoprotein $\mathrm{gB}$ seems to regulate viral surfing, as it binds to the HS expressed on the filopodia. Once the virus is bound, it travels unilaterally to the cell surface, where $\mathrm{gD}$ proceeds to bind with one of its four receptors (discussed below) and the process of virus penetration and membrane fusion ensues [14, 16-18].

\section{GLYCOPROTEINS AND RECEPTORS IMPORTANT FOR ENTRY}

The glycoproteins $\mathrm{gB}$ and $\mathrm{gH}$ play a significant role in membrane fusion, allowing the lipids in both the viral and cell membranes to mix. Of all the glycoproteins involved in entry, $\mathrm{gB}$ is the most conserved and is likely to function as a membrane fusogen [19]. As a fusogenic protein it is quite unique and has not been classified as either a class I or class II fusion protein. In fact, it contains characteristics of both classes; namely, an $\alpha$-helical coiled-coil core and extended $\beta$ hairpins [19]. In addition, multiple domains play crucial roles in the functioning of $\mathrm{gB}$ [20]. Without $\mathrm{gL}, \mathrm{gH}$ is only poorly expressed at the cell surface and it may be structurally immature [21, 22]. gL, therefore, has been implicated as a kind of guide for $\mathrm{gH}$, so that the latter is able to carry out its function in cell entry [22]. The main purpose of $\mathrm{gD}$ is to bind to a receptor on the cell surface to induce viral fusion to the cell membrane [2, 23]. At the core of $\mathrm{gD}$ is a $\mathrm{V}$-shaped immunoglobulin domain and its flexible $\mathrm{N}$-terminus folds into a hairpin-like structure. Several residues on the Cterminus of $\mathrm{gD}$ regulate receptor binding [23, 24].

$\mathrm{gD}$ can use three different families of receptors to enter into uninfected cells (Table 1). Herpesvirus entry mediator, or HVEM, is one such receptor that aids in entry along with its normal function within the tumor necrosis factor receptor (TNFR) superfamily as a part of the process of apoptosis. Normally, HVEM binds to LIGHT, a member of the tumor necrosis factor (TNF) superfamily that activated $\mathrm{T}$ cells express [25]. Nectin-1 and nectin-2, affiliated with the immunoglobulin (Ig) family, have also been shown to act as receptors [2]. Generally, these relatives of the poliovirus receptor play a role in cell adhesion. While four nectins have been discovered, only the aforementioned two can act as receptors for HSV gD [26]. Finally, 3-O sulfated heparan sulfate (3-OS-HS), a polysaccharide belonging to the HS family and thus consisting of a basic structure of repeating disaccharide units, can be used for entry [27-30]. However, only specific isoforms of 3-OS-HS, resulting from the action of certain 3-O-sulfotransferases, are able to bind to gD [27].

The receptors have been found to be specific to certain types of cells. For example, nectin- 1 is generally used by herpesviruses entering epithelial cells [2]. In activated $\mathrm{T}$ lymphocytes or Trabecular meshwork cells of the eye, HVEM is the major receptor [26, 31]. In addition, the type of virus can influence the receptor used. Nectin-2 has not 


\section{A. HSV Genome}

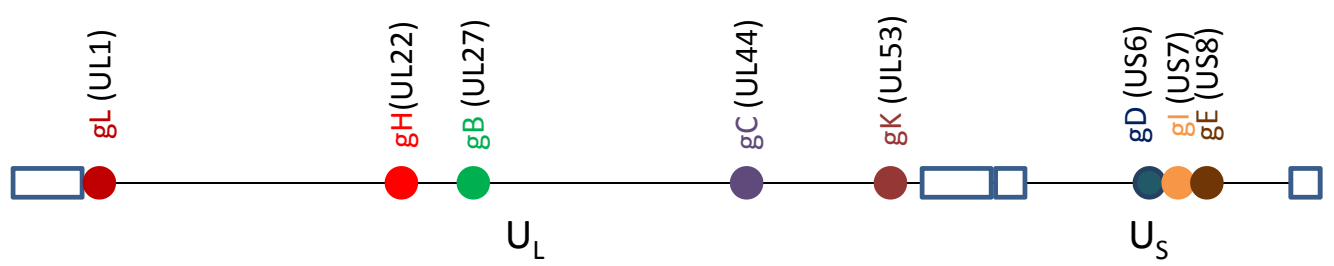

\section{B. Pathways of HSV entry into a host cell}

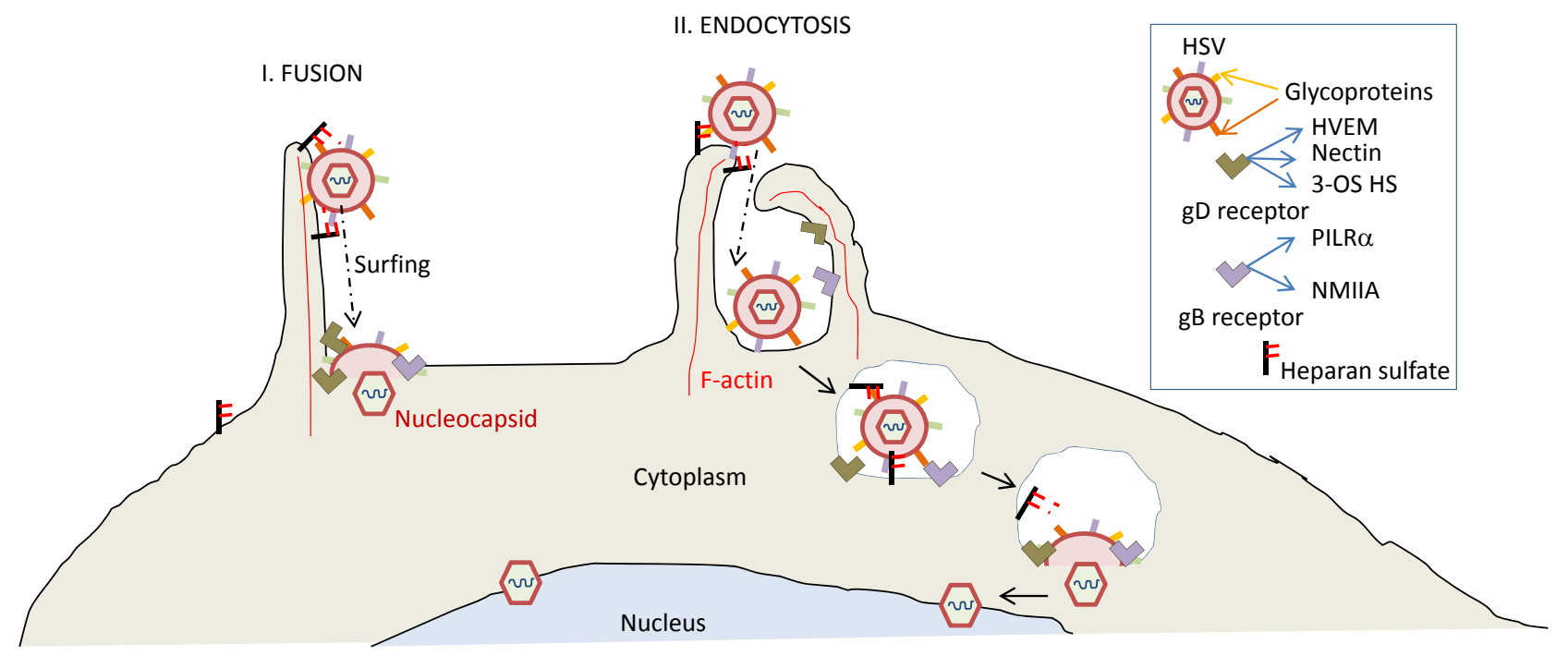

Fig. (1). Genomic distribution of HSV glycoproteins involved in viral entry and cell-to-cell spread. A. Glycoprotein genes required for HSV1 entry and spread. HSV contains a 152-kb double-stranded linear DNA genome encoding over 80 viral genes that are arranged as unique long (UL) and unique short (US) segments flanked by inverted repeat sequences (boxes). The inverted repeats contain sequences required for cleavage/packaging of the viral genome. The genes encoding glycoproteins important for HSV-1 entry and spread are shown. B. Pathways of HSV entry into cells. Two major pathways of entry (I) penetration by viral envelope fusion at the plasma membrane and (II) endocytosis are shown.

shown significant results in helping wild-type HSV-1 entry, while HSV-2 does not seem to use 3-OS HS [1, 26]. gB, on the other hand, can use paired immunoglobulin-like receptor alpha (PILR- $\alpha$ ) as a coreceptor for cell entry [32]. While both types of the herpesvirus may use this receptor, it has been proved ineffectual for HSV-2 [33]. In addition, the binding of $\mathrm{gB}$ to PILR- $\alpha$ may aid in evading the immune system by sending inhibitory signals to the host cell. Recently gB was also shown to bind non-muscle myosin heavy chain IIA [34]; however, a clear significance of this interaction is not well established. Furthermore, similar to $\mathrm{gB}, \mathrm{gH}$ also has two receptors that may aid in entry. B5, which is a type 2 membrane protein that is able to form an $\alpha-$ helix for coiled-coils, can interact with other proteins containing $\alpha$-helices such as HSV-1 gH, which may help it facilitate HSV-1 fusion [35]. The functional effect on HSV entry of the second possible receptor for $\mathrm{gH}, \alpha \mathrm{v} \beta 3$ integrins, has not yet been well understood [36].

Likewise, very limited information is available on the role of entry receptors in HSV-1 endocytosis [37]. Some
Table 1. Cell Lines with Known gD Receptors

\begin{tabular}{|c|c|}
\hline Cell Line (Virus) & $\begin{array}{c}\text { Major Entry } \\
\text { Receptor }\end{array}$ \\
\hline \hline Human conjunctival epithelium (HSV-1) & Nectin-1, HVEM \\
\hline Human corneal fibroblasts, primary (HSV-1) & 3-OS HS \\
\hline Human corneal fibroblasts, primary (HSV-2) & HVEM \\
\hline Human corneal epithelium (HSV-1) & Nectin-1 \\
\hline Human Mesenchymal Stem Cells (HSV-1) & 3-OS HS \\
\hline Human Trabecular Meshwork Cells, primary (HSV-1) & HVEM \\
\hline HeLa Cells (HSV-1, HSV-2) & Nectin-1, HVEM \\
\hline Retinal pigment epithelial cells (HSV-1) & Nectin-1 \\
\hline Retinal pigment epithelial cells (HSV-2) & Nectin-1 \\
\hline Radial glial cells and Cajal-Retzius cells & Nectin-1 \\
\hline Neuronal cells (HSV-1, HSV-2) & Nectin-1 \\
\hline
\end{tabular}


recent studies have suggested that HSV-1 can induce primary human corneal fibroblasts to engulf it in a form of an atypical endocytosis. Because this method may not be $\mathrm{pH}$ dependent and does not involve the use of clathrin-coated vesicles or caveolae, the uptake of HSV into cells most closely resembles phagocytosis [15]. This method may also need actin rearrangement and dynamin assembly to function, as well as the work of RhoA, a member of the Rho GTPase family, and some PI3 kinases [15, 38]. As aforementioned, HSV-1 induces the formation of protrusions from the cell surface. Association with these protrusions, as well as the clustering of $\mathrm{gD}$ receptors in large vesicles, marks this form of uptake [15]. It is quite likely that the virus then fuses with the membrane of an intra-cellular vesicle to release its capsid into the cytosol [15]. Recently, it was demonstrated that virus-cell fusion is also facilitated by syndecan-1 and syndecan-2 proteoglycan core proteins, as it was found that down-regulation of syndecan-1 and syndecan-2 led to a statistically significant decrease in HSV-1 entry and membrane fusion [39]. Since syndecans have been implicated in endocytosis [40] it is possible that they help with the virion fusion at a vesicular membrane. HSV-1 internalization via endosomes may allow virus to enter by both ways, fusion of the viral envelope at the plasma membrane and also by endocytosis. HSV-1 shows a very broad host range and quite possibly, it may have evolved versatile ways to enter into its multiple host cell-types.

Post fusion, the nucleocapsid and tegument proteins of the virus are released into the cytoplasm of the cell through the fusion pore. They are aided in their travel to the nucleus by the dynein-dynactin protein complex, which some viral tegument proteins may also take part in. The viral capsid contains VP26, which interacts with two dynein light chains: Tctex 1 and rp3. VP26 attracts dynein to the viral capsid, which then transports the virus along the microtubules (MT) of the cell [41]. The virus is taken first to the microtubule organizing center (MTOC) and then to the nucleus itself, where it binds to the nuclear pore complexes (NPCs). The viral DNA is released through these pores into the nucleus, where viral replication will occur [41].

\section{CELL-TO-CELL SPREAD}

HSV-1 has the ability to use cell-to-cell spread to transfer the virus between infected and uninfected cells. Rather than using incessant infection of host epithelial and lymphocytic cells to reproduce for more infection, cell-to-cell spread uses various methods, including cell-to-cell fusion, virion fusion across tight junctions, transfer across synapses and the recruitment of actin-containing structures to spread the virus directly from an infected to an uninfected cell. Proposals for how these phenomena occur have been supported by studies aimed to identify molecular details of the events.

Cell-to-cell fusion involves the fusion of plasma membranes of infected and uninfected cells to form a syncytial cell. However, a "microfusion" can occur where the two cells fuse but remain structurally independent. Theoretically, any cell with machinery needed for virus-cell fusion can undergo cell-to-cell fusion with an infected cell. The binding of HSV glycoprotein $\mathrm{gD}$ on an infected cell to its receptors on uninfected cells is essential to both virus-cell and cell-to-cell fusion [42-44]. In addition to $\mathrm{gB}, \mathrm{gH}-\mathrm{gL}$, other glycoproteins including $\mathrm{gE}$ and $\mathrm{gL}$, have been found as essential to the process, as $\mathrm{gE}$ and $\mathrm{gL}$ form a heterodimer in the trans-Golgi network (TGN) necessary for fusion of epithelial cells, which can be halted by a disruption of early sorting through the TGN [45]. Additionally, gK was found to be a vital component of cell-to-cell fusion in corneal and trigeminal ganglia cells, as mice infected with HSV-1 lacking $\mathrm{gK}$ displayed a significant decrease in cell-to-cell fusion [46].

Several $\mathrm{gD}$ receptors have been shown to play roles in cell-to-cell fusion, including HVEM, nectin-1 and 3-OS-HS. Tiwari et al. found 3-O sulfated heparan sulfate to mediate cell-to-cell fusion, as cells lacking the receptor showed a significant decrease in cell-to-cell fusion [47]. O'Donnell \& Shukla recently found heparan sulfate to have a regulatory role on cell-to-cell fusion in HeLa cells [48]. Involvement of syndecans in cell-to-cell spread has also been reported [39]. Although little is known on conformational changes that occur before and during fusion, unpublished data shows the extension of an arm towards the uninfected cell as a means of starting cell-to-cell fusion (O'Donnell and Shukla, unpublished results). Currently, very limited information is available on the structural details of cell fusion and involvement of heavy changes in cytoskeleton is highly suspected [15].

Cell-to-cell fusion is extremely advantageous for HSV-1. Because HSV-1 replicates in the nucleus, syncytial cells, which contain multiple nuclei, formed after fusion allow increase in virus reproduction and spread. Additionally, syncytial cellsallow HSV-1 to avoid innate and adaptive immune defenses against the virus, which range from the innate mediators of mucosal immunity to antibodies and phagocytes that target HSV-1. However, cell-to-cell fusion poses few disadvantages, which include the lack of the ability to easily spread throughout various parts of the body. Normally, the release of newly replicated viruses in blood would allow them to quickly travel to the entire body, HSV1 is only rarely found in the blood $[1,42]$.

\section{CELL JUNCTIONS AND SYNAPSES IN HSV-1 SPREAD}

HSV-1 can also move across intercellular junctions, which seal adjacent epithelial cells together to prevent movement of small molecules and particles across barriers. Several factors have been found to play roles in the movement of HSV-1 across tight junctions, including glycoproteins $\mathrm{gB}, \mathrm{gD}, \mathrm{gH}-\mathrm{gL}$ and $\mathrm{gE}-\mathrm{gI}$ [42]. HSV-1 can bind to complexes involved in cell junctions, including nectin-1, an entry receptor for HSV-1 and major component of adherens junctions [49]. gE-gI possibly facilitates the attachment of HSV-1 to intercellular junctions, as the accumulation of gE-gI was observed to colocalize with junction proteins including e-cadherins of human epithelial cells, while it did not accrue at lateral surfaces that were not in contact with other cells [50]. Because movement across tight junctions is a form of cell-to-cell fusion, advantages provided by this type of cell-to-cell spread include the ability to avoid neutralizing antibodies and other innate and adaptive immune defenses. The main disadvantage is that cell-to-cell spread may be slower than the transport via blood to various parts of the body. 
HSV-1 can be transferred across synapses of neurons, a process which is not completely understood. $\mathrm{gD}, \mathrm{gB}$ and $\mathrm{gH}-\mathrm{gL}$ are always necessary for trans-neuronal spread of HSV-1. The virus can travel in an anterograde movement towards axon termini in an infected neuron and be transferred across synaptic space to other cells $[27,42]$. It is believed that $\mathrm{gD}$ induces the formation of varicosities on neurons and can be transferred between neurons or from the neuron to a non-neuronal cell. De Regge et al. found that the addition of a soluble form of PRV$\mathrm{gD}$ in neurons infected with pseudorabies virus (PRV), a member of the $\alpha$ - herpesvirus family, resulted in the formation of varicosities in 60-70 percent of axons [51]. Also, nectin-1 was found to play a role in the formation of varicosities, as the addition of the antibody CK6, which binds the ectodomain of nectin-1, resulted in the formation of varicosities on 70 percent of cells [51]. The roles of $\mathrm{gD}$ and nectin-1 in the formation of varicosities in PRV- infected cells suggests that they may also play a role in the formation of varicosities in HSV-infected cells, promoting the spread of the virus from neurons to other cells.

HSV-1 can also recruit actin-containing structures for its spread from cell-to-cell. Actin-containing membrane processes project out from the cell, contacting neighboring cells and allowing the movement of the virus from the protrusions to neighboring cells. It has been found that the viral tegument protein VP-16 accumulated in long protrusions from infected cells and that these protrusions fused with neighboring cells to allow the transfer of the virus from the infected to uninfected cell [52]. The authors suggested that these protrusions may have been related to actin-containing structures, which would indicate that VP-16 may play a role in the extension of these structures towards neighboring cells.

Because HSV-1 infects epithelial, and neuronal cells including those in the eyes [53], cell-to-cell spread is critical for its survival and ability to reach the trigeminal ganglia for the establishment of latency. Epithelial cells and innervating nerve endings usually are in close proximity with one another, increasing the chances of cell-to-cell spread. Also, close proximity increases chances of the formation of tight junctions and infection of neurons encourages cell-to-cell spread through transfer across synapses [27]. The whole thing is also likely to be true with HSV-2 as well, since both are highly homologous neurotropic viruses that commonly share entry receptors and other cellular mediators of virus spread including cellular motors for transport along the microtubules.

General advantages of cell-to-cell spread include evasion of both innate and adaptive immune responses, which range from the body's physical barriers to phagocytes. For example, HSV-1 could infect mucosal cells, while it could not penetrate the high viscosity mucosal membranes without cell-to-cell spread. Other advantages include the speed in which cells are infected after viral replication. Following a normal route of infection, newly replicated viruses would exit the cell until locating new host cells, a process which can consume large amounts of time, making the virus increasingly susceptible to immune defense mechanisms. As stated earlier, an advantage of the formation of a syncytium with multiple nuclei is advantageous for HSV-1, as it HSV-1 replicates in nuclei of host cells. Overall, cell-to-cell spread, through membrane fusion, transfer across synapses, recruitment of actin-containing structures, or movement across tight junctions, provides numerous advantages to HSV-1, promoting pathogenesis of the virus. It may also represent one of the strongest aspects of HSV-1 lifecycle that give the virus the ability to survive for the lifetime of its host.

\section{CONCLUSION}

HSV can infect a wide variety of host cell types. One important reason behind this is the versatile ability of the virus to use multiple receptors and pathways for entry. Entry is also perhaps the most critical step in viral lifecycle as it allows the virus to initiate an infection that lasts for the lifetime of its host. Regardless of HSV entry receptors or pathways utilized, the initial infection almost always results in the establishment of latency. Recent advances in the field of HSV cellular receptors and HSV entry glycoproteins' structures, interactions and functions have broadened our understanding of the pathogen. We are now much more knowledgeable about the identity of HSV entry receptors. However, more progress is needed on specific contributions of entry receptors, role of intracellular signaling in HSV entry, and characterization of molecular mechanisms that allow the virus to successfully evade host's immune system. Such knowledge will definitely help with the development of new and potent HSV vaccines and anti-HSV drugs. It is important that the huge prevalence of HSV in the human population worldwide be controlled. Failing to do so will continue to have strong implications for not just acquiring the HSV-related diseases but also additional viral diseases, which may be facilitated by a prior infection with HSV.

\section{ACKNOWLEDGEMENTS}

This work was supported by NIH grants AI057860 (D.S), AI081869 (D.S.) and a Core Grant EY01792. D.S. is a recipient of the Lew Wasserman Merit award from Research to Prevent Blindness, Inc. (RPB).

\section{REFERENCES}

[1] Shukla D, Spear PG. Herpesviruses and heparan sulfate: an intimate relationship in aid of viral entry. J Clin Invest 2001; 108(4): 503-10.

[2] Akhtar J, Shukla D. Viral entry mechanisms; cellular and viral mediators of herpes simplex virus entry.FEBS J 2009; 276: 7228-36.

[3] Esmann J. The many challenges of facial herpes simplex virus infection. J Antimicrob Chemother 2001; 47(1): 17-27.

[4] Kaufman HE, Azcuy AM, Varnell ED, Sloop GD, Thompson HW \& Hill JM. HSV-1 DNA in tears and saliva of normal adults. Invest Ophthalmol Vis Sci 2001; 46: 241-7.

[5] Toma HS, Murina AT, Areaux RG Jr., et al. Ocular HSV-1 latency, reactivation and recurrent disease. Semin Ophthalmol 2008; 23(4): 24973.

[6] Wald A, Koelle DM, Fife K, et al. Safety and immunogenicity of long HSV-2 peptides complexed with rhHsc70 in HSV-2 seropositive persons. Vaccine 2011. [Epub ahead of print].

[7] Schacker T, Ryncarz AJ, Goddard J, Diem K, Shaughnessy M, Corey L. Frequent recovery of HIV-1 from genital herpes simplex virus lesions in HIV-1 infected men. J Am Med Asso 1998; 280(1): 61-6.

[8] Cooper GP, Kaplan DW. Genital herpes. CRS - Adult Health Advisor 2009; 1 .

[9] Jones C. Vertical transmission of genital herpes. Drugs 2009; 69(4): 421-34.

[10] Kesson A. Management of neonatal herpes simplex virus infection. Peadiatr Drugs 2001; 3(2): 81-90.

[11] Awasthi S, Lubinski J, Friedman H. Immunization with HSV-1 glycoprotein $\mathrm{C}$ prevents immune evasion from complement and enhances the efficacy of an HSV-1 glycoprotein D subunit vaccine. Vaccine 2009; 27(49): 6845-53.

[12] Orr MT, Edelmann KH, Vieira J, Corey L, Raulet DH, Wilson CB. Inhibition of MHC class II is a virulence factor in herpes simplex virus infection. PLoS Pathog 2005; 1(1): e7. 
[13] Melchjorsen J, Matikainen S \&Paludan S. Activation and evasion of innate antiviral immunity by herpes simplex virus. Viruses 2009; 1(3): 737-59.

[14] Oh MJ, Akhtar J, Desai P, Shukla D. A role for heparan sulfate in viral surfing. Biochem Biophys Res Commun 2010; 391(1): 176-81.

[15] Clement C, Tiwari V, Scanlan PM, Valyi-Nagy T, Yue BY, Shukla D. A novel role for phagocytosis-like uptake in herpes simplex virus entry. J Cell Bio 2006; 174: 1009-21.

[16] Ladwein M, Rottner K. On the Rho'd: the regulation of membrane protrusions by Rho-GTPases. FEBS Lett 2008; 582(14): 2066-74.

[17] Dixit R, Tiwari V, Shukla D. Herpes simplex virus type 1 induces filopodia in differentiated P19 neural cells to facilitate viral spread. Neurosci Lett 2008; 440: 113-8.

[18] Akhtar J, Tiwari V, Oh MJ, et al. HVEM and nectin-1 are the major mediators of herpes simplex virus 1 (HSV-1) entry into human conjunctival epithelium. Invest Ophthalmol Vis Sci 2008; 49(9): 402635 .

[19] Heldwein EE, Lou H, Bender FC, Cohen GH, Eisenberg RJ, Harrison SC. Crystal structure of glycoprotein B from herpes simplex virus 1 2006; 313: 217-20.

[20] Bender FC, Samanta M, Heldwein EE, et al. Antigenic and mutational analyses of herpes simplex virus glycoprotein B reveal four functional regions. J Virol 2007; 81: 3827-41.

[21] Scanlan PM, Tiwari V, Bommireddy S, Shukla D. Cellular expression of gH confers resistance to herpes simplex virus type-1 entry. Virology 2003; 312(1): 14-24.

[22] Peng T, Ponce-de-Leon M, Jiang H, et al. The gH-gL complex of herpes simplex virus (HSV) stimulates neutralizing antibody and protects mice against HSV type 1 challenge. J Virol 1998; 72(1): 65-72.

[23] Connolly SA, Landsburg DJ, Carfi A, Wiley DC, Eisenberg RJ, Cohen, GH. Structure-based analysis of the herpes simplex virus glycoprotein D binding site present on herpesvirus entry mediator HveA (HVEM). J Virol 2002; 76: 10894-904.

[24] Krummenacher C, Supekar VM, Whitbeck JC, et al. Structure of unliganded HSV $\mathrm{gD}$ reveals a mechanism for receptor-mediated activation of virus entry. EMBO J 2005; 24(23); 4144-53.

[25] Mauri DN, Ebner R, Montgomery RI, et al. LIGHT, a new member of the TNF superfamily, and lymphotoxin [alpha] are ligands for herpesvirus entry mediator. Immunity 1998; 8: 21-30.

[26] Spear PG. Herpes simplex virus: receptors and ligands for cell entry. Cell Microbiol 2004; 6: 401-10.

[27] Shukla D, Liu J, Blaiklock P, et al. A novel role for 3-O-sulfated heparan sulfate in herpes simplex virus 1 entry. Cell 1999; 99(1): 13-22.

[28] Tiwari V, O'Donnell CD, Oh MJ, Valyi-Nagy T, Shukla D. A role for 3-O-sulfotransferase isoform-4 in assisting HSV-1 entry and spread. Biochem Biophys Res Commun 2005; 338(2); 930-7.

[29] O'Donnell CD, Tiwari V, Oh MJ, Shukla D. A role for heparan sulfate 3-O-sulfotransferase isoform 2 in herpes simplex virus type 1 entry and spread. Virology 2006; 346(2): 452-9.

[30] Xia G, Chen J, Tiwari V, et al. Heparan sulfate 3-O-sulfotransferase isoform 5 generates both an antithrombin-binding site and an entry receptor for herpes simplex virus, type 1 . J Biol Chem 2002; 277(40): $37912-9$

[31] Tiwari V, Clement C, Scanlan PM, Kowlessur D, Yue BY, Shukla D. A role for herpesvirus entry mediator as the receptor for herpes simplex virus 1 entry into primary human trabecular meshwork cells. J Virol 2005; 79(20): 13173-9.

[32] Satoh T, Arii J, Suenaga T, et al. PILRalpha is a herpes simplex virus-1 entry coreceptor that associates with glycoprotein B. Cell 2008; 132(6): 935-44.

[33] Shukla SY, Singh YK, Shukla D. Role of nectin-1, HVEM, and PILRalpha in HSV-2 entry into human retinal pigment epithelial cells. Invest Ophthalmol Vis Sci 2009; 50(6): 2878-87.
[34] Arii J, Goto H, Suenaga T, et al. Non-muscle myosin IIA is a functional entry receptor for herpes simplex virus-1. Nature 2010; 467(7317): 85962

[35] Perez A, Li QX, Perez-Romero P, et al. A new class of receptor for herpes simplex virus has heptad repeat motifs that are common to membrane fusion proteins. J Virol 2005; 79(12): 7419-30.

[36] Parry C, Bell S, Minson T, Browne H. Herpes simplex virus type 1 glycoprotein $\mathrm{H}$ binds to alphavbeta3 integrins. J Gen Virol 2005; 86(Pt 1): 7-10.

[37] Nicola AV, McEvoy AM, Straus SE. Roles for endocytosis and low $\mathrm{pH}$ in herpes simplex virus entry into HeLa and Chinese hamster ovary cells. J Virol 2003; 77(9): 5324-32.

[38] Tiwari V, Shukla D. Phosphoinositide 3 kinase signalling may affect multiple steps during herpes simplex virus type-1 entry. J Gen Virol 2010; 91(Pt 12): 3002-9.

[39] Basca S, Karasneh G, Dosa S, Liu J, Valyi-Nagy T, Shukla D. Syndecan-1 and syndecan-2 play key roles in herpes simplex virus type-1 infection. J Gen Virol 2011; 92: 1-11.

[40] Foley EM, Esko JD. Hepatic heparan sulfate proteoglycans and endocytic clearance of triglyceride-rich lipoproteins. Prog Mol Biol Transl Sci 2010; 93: 213-33.

[41] Douglas MW, Diefenbach RJ, Homa FL, et al. Herpes simplex virus type 1 capsid protein VP26 interacts with dynein light chains RP3 and Tctex 1 and plays a role in retrograde cellular transport. J Biol Chem 2004; 279(27): 28522-30.

[42] Browne H, Bruun B, Minson T. Plasma membrane requirements for cell fusion induced by herpes simplex virus type 1 glycoproteins $\mathrm{gB}$, gD, gH and gL. J Gen Virol 2001; 82: 1419-22.

[43] Pertel EP, Fridberg A, Parish LM, Spear GP. Cell fusion induced by herpes simplex virus glycoproteins $\mathrm{gB}, \mathrm{gD}$, and $\mathrm{gH}-\mathrm{gL}$ requires a $\mathrm{gD}$ receptor but not necessarily heparan sulfate. Virology 2001; 279(1): 313-24

[44] Atanasiu D, Saw W, Cohen G, Eisenberg R. Cascade of events governing cell-cell fusion induced by herpes simplex virus glycoproteins gD, gH/gL, and gB. J Gen Virol 2010; 84(23): 12292-9.

[45] Potter BW. An overview of ocular herpetic disease. Rev Optometry 2010; 76(8): 76-83

[46] David AT, Baghian A, Foster TP, Chouljenko VN \& Kousoulas KG. The herpes simplex virus type 1 (HSV-1) glycoprotein $\mathrm{K}(\mathrm{gK})$ is essential for viral corneal spread and neuroinvasiveness. Curr Eye Res 2008; 33(5): 455-67.

[47] Tiwari V, Clement C, Duncan MB, Chen J, Liu J, Shukla D. A role for 3-O-sulfated heparan sulfate in cell fusion induced by herpes simplex virus type 1. J Gen Virol 2004; 85(4): 805-9.

[48] O'Donnell C, Shukla D. A novel function of heparan sulfate in the regulation of cell-cell fusion. J Biol Chem 2009; 284(43): 29654-65.

[49] Spear PG. Viral interactions with receptors in cell junctions and effects on junctional stability. Dev Cell 2002; 3(4): 462-4.

[50] Dingwell KS, Johnson DC. The herpes simplex virus gE-gI complex facilitates cell-cell spread and binds to components of cell junctions. J Virol 1998; 72(11): 8933-42.

[51] De Regge N, Nauwynck HJ, Geenen K, et al. Herpesvirus Glycoprotein $\mathrm{D}$ interaction with sensory neurons triggers formation of varicosities that serve as virus exit sites. J Cell Biol 2006; 174(2): 267-75.

[52] La Boissière $\mathrm{S}$, Izeta $\mathrm{A}$, Malcomber $\mathrm{S}$, O'Hare $\mathrm{P}$ Compartmentalization of VP16 in cells infected with recombinant herpes simplex virus expressing VP16-green fluorescent protein fusion proteins. J Virol 2004; 78(15): 8002-14.

[53] Tiwari V, Oh MJ, Kovacs M, Shukla SY, Valyi-Nagy T, Shukla D. Role for nectin-1 in herpes simplex virus 1 entry and spread in human retinal pigment epithelial cells. FEBS J 2008; 275(21): 5272-85. 